\title{
Generalized variation and translation operator in some sequence spaces
}

\author{
J. MUSIELAK and A. WASZAK \\ (Received August 5, 1987, Revised February 23, 1988)
}

\begin{abstract}
There are defined and investigated some spaces of sequences provided with two-modular structure given by generalized variations and the translation operator. The results are applied to obtain an approximation theorem by means of translated sequences.

1. Let $x=\left(t_{i}\right)=\left(t_{i}\right)_{i=0}^{\infty}$ be a sequence of real numbers. We denote also $(x)_{j}=t_{j}$ for $j=0,1,2, \ldots$ We introduce two auxiliary notations: this of the $\Phi$-variation of $x$ and that of the sequential modulus of $x$.

1. 1. Let $X$ be the space of all real sequences and let $\Phi$ be a $\varphi$-function (see e.g. [4], 1.9). The $\Phi$-variation $w_{\Phi}(x)$ of $x \in X$ is defined as

$$
w_{\Phi}(x)=\sup _{\left(n_{i}\right)} \sum_{i=1}^{\infty} \Phi\left(\left|t_{n_{i}}-t_{n_{i-1}}\right|\right),
$$
\end{abstract}

where the supremum runs through all increasing subsequences $\left(n_{i}\right)$ of indices (see [2]). $w_{\Phi}$ is a pseudomodular in $X$ defining the modular space

$$
X_{\Phi}=X_{w_{\Phi}}=\left\{x \in X: w_{\Phi}(\lambda x) \longrightarrow 0 \text { as } \lambda \longrightarrow 0_{+}\right\}
$$

(see [7], [5] and also [8]). $\|\cdot\|_{\Phi}$ will denote the Luxemburg pseudonorm in $X_{\Phi}$ (see [4]). It is easily seen that $X_{\Phi} \subset c$, where $c$ is the space of convergent sequences, and $X_{\Phi}$ is strongly modular complete and complete in the norm (see [2] and [5]).

1.2. Given any sequence $x=\left(t_{i}\right)_{i=0}^{\infty}$, we write

$$
\left(\tau_{m} x\right)_{j}=\left\{\begin{array}{l}
t_{j} \text { for } j<m, \\
t_{m+j} \text { for } j \geq m,
\end{array}\right.
$$

where $m, j=0,1,2, \ldots$ (see [3], also [4], 7.17). The sequence $\tau_{m} x=$ $\left(\left(\tau_{m} x\right)_{j}\right)_{j=0}^{\infty}$ is called the $m$-translation of the sequence $x$.

1.3. The sequential modulus of the sequence $x=\left(t_{i}\right)_{i=0}^{\infty}$ is defined as

$$
\omega(x, r)=\sup _{m \geq r} \sup _{i}\left|\left(\tau_{m} x\right)_{i}-t_{i}\right|
$$

where $r=0,1,2, \ldots$ Obviously, we have

$$
\omega(x, r)=\sup _{m \geq r} \sup _{i \geq m}\left|t_{m+i}-t_{i}\right|
$$


for $r=0,1,2, \ldots$.

For example, taking $x=\left(a^{i}\right)_{i=0}^{\infty}$ with $0<a<1$ or $x=\left(\frac{1}{i+1}\right)_{i=0}^{\infty}$ or $x=$ $\left(1+\frac{1}{2}+\cdots+\frac{1}{i+1}\right)_{i=0}^{\infty}$, then we have $\omega(x, r)=a^{r}\left(1-a^{r}\right)$ for $r \geq-\ln 2 / \ln a$ or $\omega(x, r)=\frac{r}{(r+1)(2 r+1)}$ for $r \geq 1$ or $\omega(x, r)=\ln 2$ for $r \geq 0$, respectively.

2. We shall consider two spaces of sequences $X(\Psi)$ and $X(\Phi, \Psi)$, defined by means of the sequential modulus and $\Phi$-variation of the sequence.

2. 1. Let $\Phi$ be a $\varphi$-function and let $\Psi$ be a nonnegative, nondecreasing function of $u \geq 0$ such that $\Psi(u) \longrightarrow 0$ as $u \longrightarrow 0_{+}$. Then we write

$$
\begin{aligned}
& X(\Psi)=\{x \in X: r \Psi(\omega(\lambda x, r)) \longrightarrow 0 \text { as } r \longrightarrow \infty \text { for a } \lambda>0\} \\
& X(\Phi, \Psi)=X_{\Phi} \cap X(\Psi) .
\end{aligned}
$$

Obviously, $X(\Psi)$ and $X(\Phi, \Psi)$ are vector spaces. If $\Psi$ satisfies the condition $\left(\Delta_{2}\right)$ for small $u \geq 0$, then one may take fixed $\lambda=1$ in the definition of $X(\Psi)$.

2.2. We define now for every $x \in X$

$$
\zeta(x)=\sup _{r} r \Psi(\omega(x, r)) \text {. }
$$

Obviously, $\zeta$ is a pseudomodular in $X$. The respective modular space will be denoted by $X_{\zeta}$; we have $X(\Phi, \Psi) \subset X(\Psi) \subset X_{\zeta}$.

Let us remark that if $\Psi$ is increasing and s-convex for $u \geq 0$ with some $0<s \leq 1$, then $\zeta$ is an $s$-convex pseudomodular in $X$ and

$$
\|x\|_{\zeta}^{s}=\sup _{r \geq 1}\left(\frac{\omega(x, r)}{\Psi^{-1}(1 / r)}\right)^{s}
$$

where $\Psi^{-1}$ is the inverse to $\Psi$, because

$$
\begin{aligned}
\|x\|_{\xi}^{s} & =\inf \left\{u>0: \zeta\left(\frac{x}{u^{1 / s}}\right) \leq 1\right\} \\
& =\inf \left\{u>0: \frac{\omega(x, r)}{u^{1 / s}} \leq \Psi^{-1}\left(\frac{1}{r}\right) \text { for all } r \geq 1\right\} .
\end{aligned}
$$

For example, taking $x=\left(a^{i}\right)_{i=0}^{\infty}, 0<a<1$ and both $\Phi, \Psi s$-convex with $0<s \leq 1$, we have $w_{\Phi}(\lambda x) \leq \Phi(\lambda)\left(1-a^{s}\right)^{-1}$ for $\lambda>0$ and $r \Psi(\omega(\lambda x, r)) \leq$ $r \Psi\left(\lambda a^{r}\right) \leq r\left(a^{s}\right)^{r} \Psi(\lambda) \longrightarrow 0$ as $r \longrightarrow \infty$. Hence $x \in X(\Phi, \Psi)$.

2.3. Let $\bar{c}$ be the space of all sequences $x=\left(t_{i}\right)_{i=0}^{\infty}$ such that $t_{0}$ and $t_{1}$ are arbitrary and $t_{i}=t_{i+1}$ for $i=1,2, \ldots$. Let $\Phi$ be a $\varphi$-function and let $\Psi$ be a nonnegative, increasing function such that $\Psi(u) \longrightarrow 0$ as $u \longrightarrow 0_{+}$. 
Then $w_{\Phi}(x)=\Phi\left(\left|t_{1}-t_{0}\right|\right)$ and $\omega(x, r)=0, \mathrm{r}=0,1,2, \ldots$ for $x \in \bar{c}$. Hence $\bar{c}$ is a vector subspace of $X(\Phi, \Psi)$ and $x \in \bar{c}$ is equivalent to $|x|_{\zeta}=0$, where $|\cdot|_{\zeta}$ is the $F$-pseudonorm generated by $\zeta$ (see [4], 1.5). Consequently, one may consider quotient space

$$
\tilde{X}_{\zeta}=X_{\zeta} / \bar{c}, \tilde{X}(\Psi)=X(\Psi) / \bar{c} \text { and } \tilde{X}(\Phi, \Psi)=X(\Phi, \Psi) / \bar{c},
$$

whose elements will be denoted by $\tilde{x}$, etc. Since $|x|_{\zeta}$ is constant in each of the classes $\tilde{x}$, we may define $|\tilde{x}|_{\zeta}=|x|_{\zeta}, x \in \tilde{x}$. In case if $\Psi$ is s-convex, $0<$ $s \leq 1$, we may define $\|\tilde{x}\|_{\xi}^{s}=\|x\|_{5}^{s}, x \in \tilde{x}$.

2.4. The following condition will be needed (see [4]) :

$(+)$ there exists a $u_{0}>0$ such that for every $\delta>0$ there is an $\eta>0$ satisfying the inequality $\Psi(\eta u) \leq \delta \Psi(u)$ for all $0 \leq u \leq u_{0}$.

In particular, every $s$-convex $\varphi$-function $\Psi, 0<s \leq 1$, satisfies $(+)$. There are $\varphi$-functions $\Psi$ not satisfying $(+)$, for example

$$
\Psi(u)= \begin{cases}0 & \text { for } u=0, \\ \frac{1}{\sqrt{-\ln u}} & \text { for } 0<u \leq \frac{1}{e}, \\ \text { arbitrary } & \text { for } u>\frac{1}{e} .\end{cases}
$$

It is easily seen that $(+)$ is equivalent to the following condition:

$(++)$ for any $u_{1}>0$ and $\delta_{1}>0$ there is an $\eta_{1}>0$ such that $\Psi(\eta u) \leq \delta_{1} \Psi(u)$ for all $0 \leq u \leq u_{1}$ and $0<\eta \leq \eta_{1}$.

2.5. THEOREM. Let $\Psi$ be an increasing, continuous function of $u \geq 0$, $\Psi(0)=0$, satisfying the condition $2.4(+)$. Then $\tilde{X}_{\zeta}$ and $\tilde{X}(\Psi)$ are Fréchet spaces with respect to the F-norm $|\cdot|_{\zeta}$.

Proof. Let $\left(\tilde{x}_{n}\right)$ be a Cauchy sequence in $\tilde{X}_{\zeta}$ and let $x_{n} \in \tilde{x}_{n}, x_{n}=$ $\left(t_{i}^{n}\right)_{i=0}^{\infty}$ be such that $t_{1}^{n}=0$ for all $n$. Let an $\varepsilon>0$ be given and let $\Psi^{-1}$ be the inverse to $\Psi$. There is an $N$ such that $\left|x_{p}-x_{q}\right|_{\zeta}<\Psi(\varepsilon)$ for $p, q>N$. Hence there exists a $u_{\varepsilon}, 0<u_{\varepsilon}<\Psi(\varepsilon)$, for which

$$
r \Psi\left(\frac{\omega\left(x_{p}-x_{q}, r\right)}{u_{\varepsilon}}\right) \leq u_{\varepsilon}
$$

for $p, q>N$ and $r=1,2, \ldots$, whence

$$
\omega\left(x_{p}-x_{q}, r\right) \leq u_{\varepsilon} \Psi^{-1}\left(\frac{u_{\varepsilon}}{r}\right) \leq u_{\varepsilon} \cdot \varepsilon<\varepsilon \Psi(\varepsilon)
$$

for $p, q>N, r \geq 1$. Thus 


$$
\left|t_{m+i}^{p}-t_{m+i}^{q}-t_{i}^{p}+t_{i}^{q}\right| \leq u_{\varepsilon} \Psi^{-1}\left(\frac{u_{\varepsilon}}{r}\right)<\varepsilon \Psi(\varepsilon)
$$

for $p, q>N, i \geq m \geq r$. Taking $r=1$ and $m=1$ we obtain

$$
\left|t_{i+1}^{p}-t_{i+1}^{q}\right| \leq\left|t_{i}^{p}-t_{i}^{q}\right|+\varepsilon \Psi(\varepsilon)
$$

for $p, q>N, i=1,2, \ldots$ Hence, because $t_{1}^{n}=0$ for all $n$, we see that $\left(t_{i}^{n}\right)_{n=0}^{\infty}$ are Cauchy sequence for $i=1,2, \ldots$ Let $t_{i}=\lim _{n \rightarrow \infty} t_{i}^{n}$ for $i=1,2, \ldots$, $t_{0}=0, x=\left(t_{i}\right)_{i=0}^{\infty}$. Taking $q \longrightarrow \infty$ in $(*)$, we have

(**) $\quad\left|t_{m+i}^{p}-t_{m+i}-t_{i}^{p}+t_{i}\right| \leq u_{\varepsilon} \Psi^{-1}\left(\frac{u_{\varepsilon}}{r}\right)$

for $p>N, i \geq m \geq r \geq 1$. Thus

$$
r \Psi\left(\frac{\omega\left(x_{p}-x, r\right)}{u_{\varepsilon}}\right) \leq u_{\varepsilon}
$$

for $p>N, r \geq 1$. We shall see that this implies $x_{p}-x \in X_{\zeta}$ for large $p$, i. e. $x \in X_{\zeta}$. Indeed, let $u_{\varepsilon}>0$ and $p>N$ be fixed and let $\delta>0$ be arbitrary. Taking $\delta_{1}=\delta / u_{\varepsilon}, u_{1}=\Psi^{-1}\left(u_{\varepsilon}\right)$ and $u=\frac{\omega\left(x_{p}-x, r\right)}{u_{\varepsilon}}$ in $2.4(++)$, we obtain for $0<\lambda \leq \frac{\eta_{1}}{u_{\varepsilon}}$

$$
r \Psi\left(\lambda \omega\left(x_{p}-x, r\right)\right)=r \Psi\left(\lambda u_{\varepsilon} \frac{\omega\left(x_{p}-x, r\right)}{u_{\varepsilon}}\right) \leq \delta_{1}
$$

uniformly with respect to $r$. Thus, $\zeta\left(\lambda\left(x_{p}-x\right)\right) \longrightarrow 0$ as $\lambda \longrightarrow 0_{+}$, i. e. $x_{p}-x$ $\in X_{\zeta}$. Moreover, $\left|x_{p}-x\right|_{\zeta} \leq u_{\varepsilon} \leq \Psi(\varepsilon)$ for $p>N$, i. e. $\left|x_{p}-x\right|_{\zeta} \longrightarrow 0$ as $p$ $\longrightarrow \infty$. Thus, $\tilde{X}_{\zeta}$ is complete.

We have still to show that $\tilde{X}(\Psi)$ is closed in $\tilde{X}_{\zeta}$ with respect to $|\cdot|_{\zeta}$. Let $\tilde{x}_{p} \longrightarrow \tilde{x}$ in $\tilde{X}_{\zeta}, \tilde{x}_{p} \in \tilde{X}(\Psi)$, and let $x_{p} \in \tilde{x}_{p}, x \in \tilde{x}$. Then for every $\lambda$ $>0$,

$$
r \Psi\left(\omega\left(\lambda\left(x_{p}-x\right), r\right)\right) \longrightarrow 0 \text { as } p \longrightarrow \infty
$$

uniformly with respect to $r$. Let us fix $\lambda>0$ and $\varepsilon>0$. There is an index $p_{0}$ such that $r \Psi\left(2 \omega\left(\lambda\left(x_{p}-x\right), r\right)\right)<\frac{1}{2} \varepsilon$ for $p \geq p_{0}$ and all $r$. We may choose an $r_{0}$ such that $r \Psi\left(2 \omega\left(\lambda x_{p_{0}}, r\right)\right)<\frac{1}{2} \varepsilon$ for all $r \geq r_{0}$. Hence

$$
r \Psi(\omega(\lambda x, r)) \leq r \Psi\left(2 \omega\left(\lambda\left(x-x_{p_{0}}\right), r\right)\right)+r \Psi\left(2 \omega\left(\lambda x_{p_{0}}, r\right)\right)<\varepsilon
$$

for $r \geq r_{0}$. This shows that $x \in X(\Psi)$, i. e. $\tilde{x} \in \tilde{X}(\Psi)$. 
We are going now to express Theorem 2.5 replacing the $F$-norm $|\cdot|_{\zeta}$ by the pseudomodular $\xi$ itself.

Let us remark that $\bar{c}=\{x \in X: \zeta(x)=0\}$. Moreover, $\xi(x)=0$ implies $\zeta(2 x)=0$ for all $x \in X$. By [1], 3.3, 3.2 and 3.5, $\tilde{\zeta}(\tilde{x})=\inf \{\zeta(y): y \in \tilde{x}\}$ is a modular in $\widetilde{X}_{\xi}$ and $\widetilde{X}_{\zeta}=X_{\xi} / \bar{c}=(X / \bar{c})_{\tilde{\xi}}$.

Let us still recall that a sequence $\left(x_{n}\right)$ is called $\zeta$-Cauchy, if there is a $k$ $>0$ such that for every $\varepsilon>0$ there exists an index $N$ for which $\zeta\left(k\left(x_{p}-x_{q}\right)\right)$ $<\varepsilon$ for all $p, q>N$. A modular space is called $\zeta$-complete, if every $\zeta$ Cauchy sequence of its elements is $\zeta$-convergent to an element of this space (see [5], 1.04).

2.6. THEOREM. Let $\Psi$ be an increasing, continuous function of $u \geq 0$, $\Psi(0)=0$, satisfying the condition $2.4(+)$. The spaces $\widetilde{X}_{\xi}$ and $\tilde{X}(\Psi)$ are $\tilde{\zeta}$-complete.

Proof is similar to that of 2.5 , and we give an outline only. Let $\tilde{x}_{n} \in$ $\tilde{X}_{\zeta}, x_{n}=\left(t_{i}^{n}\right)_{i=0}^{\infty} \in \tilde{x}_{n}, t_{1}^{n}=0$ for all $n$, and let $\left(\tilde{x}_{n}\right)$ be $\tilde{\zeta}$-Cauchy in $\tilde{X}_{\zeta}$. For every $\varepsilon>0$ there exists an $N$ such that $\tilde{\zeta}\left(2 k\left(\tilde{x}_{p}-\tilde{x}_{q}\right)\right)<\varepsilon$ for $p, q>N, k>$ 0 being fixed. There exists a $y \in 2 k\left(\tilde{x}_{p}-\tilde{x}_{q}\right)$ such that $\zeta(y)<\varepsilon$. Let us remark that if $z_{1}, z_{2} \in X_{\zeta}, z_{1}-z_{2} \in \bar{c}$, then

$$
\zeta\left(z_{2}\right) \leq \zeta\left(2 z_{1}\right)+\zeta\left(2\left(z_{2}-z_{1}\right)\right)=\zeta\left(2 z_{1}\right) .
$$

Taking $z_{1}=\frac{1}{2} y, z_{2} k\left(x_{p}-x_{q}\right)$, we thus have $\zeta\left(k\left(x_{p}-x_{q}\right)\right) \leq \zeta(y)<\varepsilon$ for $p, q$ $>N$. Hence $\omega\left(k\left(x_{p}-x_{q}\right), r\right)<\Psi^{-1}(\varepsilon / r)$ for $p, q>N$ and all $r$. Arguing as in the proof of 2.5 , we obtain inequalities $(*)$ and $(* *)$ with right-hand side changed to $\frac{1}{k} \Psi^{-1}(\varepsilon / r)$, which gives $r \Psi\left(\omega\left(k\left(x_{p}-x\right), r\right)\right)<\varepsilon$ for $p>N$ and every $r \geq 1$. This implies $x_{p}-x \in X_{\zeta}$, as in the proof of 2. 5. Moreover, $x_{p}$ $\in X_{\xi}$, and so $x \in X_{\xi}$. Further, $\zeta\left(k\left(x_{p}-x\right)\right) \leq \varepsilon$ for $p>N$. This implies that $\left(x_{n}\right)$ is $\zeta$-convergent to $x$. We have $\tilde{\zeta}\left(k\left(\tilde{x}_{p}-\tilde{x}\right)\right)=\inf \left\{\zeta(y): y \in k\left(\tilde{x}_{p}\right.\right.$ $-\tilde{x})\} \leq \zeta\left(k\left(x_{p}-x\right)\right) \leq \varepsilon$ for $p>N$. Thus, $\left(\tilde{x}_{n}\right)$ is $\tilde{\zeta}$-convergent to $\tilde{x}$. Consequently, $\tilde{X}_{\zeta}$ is $\tilde{\zeta}$-complete. Finally, we prove $\tilde{X}(\Psi)$ to be $\tilde{\zeta}$-closed in $\tilde{X}_{\xi}$. Let $\tilde{x}_{p} \in \tilde{X}(\Psi), \tilde{x}_{p} \stackrel{\tilde{\xi}}{\longrightarrow} \tilde{x}$. Then $\tilde{x} \in \tilde{X}_{\xi}$ and $\tilde{\zeta}\left(2 \lambda\left(\tilde{x}_{p}-\tilde{x}\right)\right) \longrightarrow 0$ as $p \longrightarrow \infty$, for some $\lambda>0$. Arguing as in the first part of the proof we obtain that $\zeta\left(\lambda\left(x_{p}-x\right)\right) \longrightarrow 0$ as $p \longrightarrow \infty$. This implies $x \in X(\Psi)$, i. e. $\tilde{x} \in \tilde{X}(\Psi)$, as in the proof of 2.5 .

3. One may ask also the question, whether Theorems 2.5 and 2.6 remain true, if we replace the space $\tilde{X}(\Psi)$ by $\tilde{X}(\Phi, \Psi)$, or equivalently, whether $\tilde{X}(\Phi, \Psi)$ is a closed subspace of $\tilde{X}(\Psi)$ with respect to the $F$-norm $|\cdot|_{\zeta}$, or the modular $\tilde{\zeta}$. A negative answer to this question is provided by 
the example $\Phi(u)=|u|, \Psi(u)=u^{2}$ and $x=\left(t_{i}\right)_{i=0}^{\infty}, x_{n}=\left(t_{i}^{n}\right)_{i=0}^{\infty}$, where $t_{i}=$ $(-1)^{i} /(i+1), \mathrm{t}_{i}^{n}=t_{i}$ for $i \leq n, t_{i}^{n}=0$ for $i>n$. Obviously, $x_{n} \in X(\Phi, \Psi), x$ $\in X(\Psi)$, but $x \notin X_{\Phi}$. This negative answer leads to putting the same question in context of two-modular convergence in $\tilde{X}(\Phi, \Psi)$.

3.1. Let us recall the notion of two-modular convergence $(\gamma$ convergence), (see [6] or [4], p. 169). Let $\left\langle X, \zeta^{\prime}, \zeta\right\rangle$ be a triple, where $\zeta^{\prime}$ and $\zeta$ are two modulars in a vector space $X$. A set $K=\left\{x \in X_{\zeta^{\prime}}: \zeta^{\prime}\left(k_{0} x\right)\right.$ $\left.\leq M_{0}\right\}$ with some $k_{0}, M_{0}>0$ is called a $\zeta^{\prime}$-ball. A sequence $\left(x_{n}\right), x_{n} \in X$ is called $\zeta^{\prime}$-bounded, if the sequence $\left(\varepsilon_{n} x_{n}\right)$ is $\zeta^{\prime}$-convergent to 0 for every sequence of numbers $\varepsilon_{n} \longrightarrow 0$. If $\left(x_{n}\right)$ is $\zeta^{\prime}$-bounded, then $x_{n} \in K, n=1$, $2, \ldots$, for some $k_{0}, M_{0}>0$ (see [6] or [4], 5.5). A sequence $\left(x_{n}\right)$ is called $\gamma$-convergent to $x, x_{n} \stackrel{\gamma}{\longrightarrow} x$, if $\left(x_{n}\right)$ is $\zeta^{\prime}$-bounded and $\zeta$-convergent to $x$. The two-modular space, i. e. the triple $\left\langle X, \zeta^{\prime}, \zeta\right\rangle$ is called $\gamma$-complete, if for every fixed $\zeta^{\prime}$-ball $K$ and every sequence $\left(x_{n}\right), x_{n} \in K$, which is $\zeta$-Cauchy, there exists an element $x \in K$ such that $x_{n} \stackrel{\gamma}{\longrightarrow} x$.

We are going now to investigate the two-modular space $<\widetilde{X}(\Phi, \Psi), \widetilde{w}_{\Phi}$, $\tilde{\zeta}>$, where $\widetilde{w}_{\Phi}(\tilde{x})=\inf \left\{w_{\Phi}(y): y \in \tilde{x}\right\}$.

Let us remark that $\widetilde{w}_{\Phi}(\tilde{x})=w_{\Phi}(\bar{x})$, where $x=\left(t_{i}\right)_{i=0}^{\infty}, \quad \bar{x}=\left(\bar{t}_{i}\right)_{i=0}^{\infty}, \bar{t}_{0}=$ $t_{1}, \bar{t}_{i} t_{i}$ for $i \geq 1$. Obviously, $\bar{x} \in \tilde{x}$, and so $\widetilde{w}_{\Phi}(\tilde{x}) \leq w_{\Phi}(\bar{x})$. Now, let $y=$ $\left(s_{i}\right)_{i=0}^{\infty} \in \tilde{x}$, then $s_{i}-t_{i}=k$ for $i=1,2, \ldots$ with some constant $k$. Denoting $\bar{y}$ $=\left(\bar{s}_{i}\right)_{i=0}^{\infty}$, where $\bar{s}_{0}=t_{1}+k, \quad \bar{s}_{i} t_{i}+k$ for $i \geq 1$, we have $w_{\Phi}(y) \geq w_{\Phi}(\bar{y})=$ $w_{\Phi}(\bar{x})$. This implies $\widetilde{w}_{\Phi}(\tilde{x}) \geq w_{\Phi}(\bar{x})$.

3.2. THEOREM. Let $\Phi$ be a $\varphi$-function and let $\Psi$ be an increasing, continuous function of $u \geq 0$, satisfying the condition $2.4(+)$ and such that $\Psi(0)=0$. Then the two-modular space

$$
<\widetilde{X}(\Phi, \Psi), \widetilde{w}_{\Phi}, \tilde{\zeta}>
$$

is $\gamma$-complete.

PROOF. Let $\widetilde{K}$ be a $\widetilde{w}_{\Phi}$-ball in $\tilde{X}\left(\underset{\zeta}{ }(\Psi)\right.$ and let $\tilde{x}_{n} \in \widetilde{K}$ for $n=1$, $2, \ldots,\left(\tilde{x}_{n}\right)$ be $\tilde{\zeta}$-Cauchy. By 2.6, $\left(\tilde{x}_{n}\right)$ is $\tilde{\zeta}$-convergent to an element $\tilde{x} \in$ $\widetilde{\mathrm{X}}(\Psi)$. Hence $\tilde{x}_{n} \stackrel{\gamma}{\longrightarrow} \tilde{x}$. We have to show that $\tilde{x} \in \tilde{K}$. It is easily seen that taking $x_{n} \in \tilde{x}_{n}, x_{n} \in X_{\Phi}$ in such a manner that the first two coordinates of $x_{n}$ are the same, we have $w_{\Phi}\left(k_{0} x_{n}\right) \leq M_{0}$ for some $k_{0}, M_{0}>0$.

Thus, writing $x_{n}=\left(t_{i}\right)_{i=0}^{\infty}$, we have

$$
\sum_{i=1}^{\infty} \Phi\left(k_{0}\left|t_{n_{i}}^{p}-t_{n_{i-1}}^{p}\right|\right) \leq M_{0}
$$

for $p=1,2, \ldots$ and any increasing sequence $\left(n_{i}\right)$ of positive integers. Since $t_{i}^{p} \longrightarrow t_{i}$ as $p \longrightarrow \infty$, where $x=\left(t_{i}\right)_{i=0}^{\infty}$, we obtain easily 


$$
\sum_{i=1}^{\infty} \Phi\left(k_{0}\left|t_{n_{i}}-t_{n_{i-1}}\right|\right) \leq M_{0}
$$

whence $w_{\Phi}\left(k_{0} x\right) \leq M_{0}$. Consequently, $\widetilde{w}_{\Phi}\left(k_{0} \tilde{x}\right) \leq M_{0}$, i. e. $\tilde{x} \in \widetilde{K}$.

4. Let $\Phi$ be a $\varphi$-function and let $\Psi$ be an increasing, continuous function for $u \geq 0$ such that $\Psi(0)=0$. We apply now the $\gamma$-convergence in $\widetilde{X}(\Phi$, $\Psi$ ) in order to obtain an approximation theorem by means of the $m$ translation, i. e. a result of the form $\tau_{m} x-x \longrightarrow 0$ in an Orlicz sequence space $1^{\Gamma}$ with a $\varphi$-function $\Gamma$ satisfying the following condition :

(i) there exist positive constants $a, b, u_{0}$ such that $\Gamma(a u) \leq b \Phi(u) \Psi(u)$ for $0 \leq u \leq u_{0}$.

It is easily seen that ( $\mathrm{i}$ ) implies, that for every $u_{1} \geq 0$ there exists a $c$ $>0$ such that $\Gamma(c u) \leq b \Phi(u) \Psi(u)$ for $0 \leq u \leq u_{1}$; indeed, if $u_{1} \leq u_{0}$ we may take $c=a$, and if $u_{1}>u_{0}$, we may put $c=a u_{0}\left(u_{1}\right)^{-1}$.

4.1. LEMMA. Let the assumptions of 4 be satisfied and let $w_{\Phi}(\lambda x)<\infty$ for $a \lambda>0$. Then

$$
\sum_{i=1}^{\infty} \Gamma\left(c \lambda\left|\left(\tau_{r} x\right)_{i}-(x)_{i}\right|\right) \leq b r \Psi(\omega(\lambda x, r)) w_{\Phi}(\lambda x)
$$

for every $r \geq 0$.

PROOF. Since $x=\left(t_{i}\right)_{i=0}^{\infty}$ is bounded, so taking $u_{1}=2 \lambda \sup _{i}\left|t_{i}\right|$, fixing $r$ and choosing $m \geq r$ arbitrarily, we obtain

$$
\begin{aligned}
\sum_{i=1}^{\infty} & \Gamma\left(c \lambda\left|\left(\tau_{m} x\right)_{i}-(x)_{i}\right|\right)=\sum_{i=m}^{\infty} \Gamma\left(c \lambda\left|t_{m+i}-t_{i}\right|\right) \\
& \leq b \Psi(\omega(\lambda x, r)) \sum_{i=m}^{\infty} \Phi\left(\lambda\left|t_{m+i}-t_{i}\right|\right) \\
& \leq b \Psi(\omega(\lambda x, r)) \sum_{k=1}^{\infty} \sum_{i=k m}^{(k+1) m-1} \Phi\left(\lambda\left|t_{m+i}-t_{i}\right|\right) \\
& \leq b \Psi(\omega(\lambda x, r)) \sum_{j=m}^{2 m-1} \sum_{k=1}^{\infty} \Phi\left(\lambda\left|t_{k m+j}-t_{(k-1) m+j}\right|\right) \\
& \leq b \Psi(\omega(\lambda x, r)) m w_{\Phi}(\lambda x) .
\end{aligned}
$$

Taking $m=r$, we get the required ineguality.

4.2. THEOREM. Let $\Phi$ and $\Gamma$ be $\varphi$-functions and let $\Psi$ be an increasing, continuous function for $u \geq 0, \Psi(0)=0$, such that 4 (i ) holds. Let $x \in \tilde{x} \in$ $\tilde{X}(\Phi, \Psi)$. Then $\tau_{r} x-x \in 1^{\Gamma}$ for all $r \geq 0$, and $\tau_{r} x-x \longrightarrow 0$ in the sense of modular convergence in $1^{\Gamma}$.

ProOF. Since $x \in X(\Phi, \Psi)$, so $w_{\Phi}(\lambda x)<\infty$ and $r \Psi(\omega(\lambda x, r)) \longrightarrow 0$ as 
$r \longrightarrow \infty$ for sufficiently small $\lambda>0$. By Lemma $4.1, \tau_{r} x-x \in 1^{\Gamma}$ for all $r \geq$ 0 . Also, taking $r \longrightarrow \infty$ in the inequality of Lemma 4 .1, we obtain $\tau_{r} x$ $-x \longrightarrow 0$ in the sense of modular convergence in $1^{\Gamma}$.

4. 3. LEMMA. Let $x_{n}=\left(t_{i}^{n}\right)_{i=0}^{\infty} \in X_{\Phi}, t_{0}^{n}=0$, for $n=1,2, \ldots$, and let $x_{n} \in K$, where $K$ is a $w_{\Phi}$-ball in $X_{\Phi}$. Then there is a constant $L>0$ such that $\left|t_{i}^{n}\right| \leq L$ for $i=0,1,2, \ldots$ and $n=1,2, \ldots$.

PROOF. Let $w_{\Phi}\left(k_{0} x_{n}\right) \leq M_{0}$ for $n=1,2, \ldots$ with some $k_{0}, M_{0}>0$, then $\Phi\left(k_{0}\left|t_{i}^{n}\right|\right)=\Phi\left(k_{0}\left|t_{i}^{n}-t_{0}^{n}\right|\right) \leq M_{0}$, and so $\left|t_{i}^{n}\right| \leq L$ for some $L>0$, because $\Phi(u) \longrightarrow \infty$ as $u \longrightarrow \infty$.

4.4. THeOREM. Let the same assumptions as in 4.3 be satisfied. Let $\tilde{x}_{n} \in \tilde{X}(\Phi, \Psi), \quad \tilde{x}_{n} \stackrel{\gamma}{\longrightarrow} 0$ in $<\tilde{X}(\Phi, \Psi), \widetilde{w}_{\Phi}, \tilde{\zeta}>$ as $n \longrightarrow \infty$ and $x_{n}\left(t_{i}^{n}\right)_{i=0}^{\infty} \in$ $\tilde{x}_{n}, t_{0}^{n}=0, t_{1}^{n}=0$ for $n=1,2, \ldots$. Then $\tau_{r} x_{n}-x_{n} \longrightarrow 0$ with respect to modular convergence in $1^{\Gamma}$ as $n \longrightarrow \infty$, uniformly for $r \geq 0$.

Proof. Since $\tilde{x}_{n} \stackrel{\gamma}{\longrightarrow} 0$, so $\tilde{x}_{n} \in \tilde{K}$, where $\tilde{K}$ is a $\widetilde{w}_{\Phi}$-ball. But $w_{\Phi}\left(k_{0} \tilde{x}_{n}\right) \leq M_{0}$ with some $k_{0}, M_{0}>0$. By Lemma $4.3,\left|t_{i}^{n}\right| \leq L$ for all $i, n$, with an $L>0$. Let $u_{1}=2 \lambda L, c=a u_{0} / u_{1}$, where $0<\lambda \leq k_{0}$. Then, by Lemma 4.1 we have

$$
\sum_{i=0}^{\infty} \Gamma\left(c \lambda\left|\left(\tau_{r} x_{n}\right)_{i}-\left(x_{n}\right)_{i}\right|\right) \leq b \zeta\left(\lambda x_{n}\right) w_{\Phi}\left(\lambda x_{n}\right) \leq b \zeta\left(\lambda x_{n}\right) M_{0} .
$$

By assumption there exists a $\lambda>0$ such that for every $\varepsilon>0$ there is an integer $N$ for which $\tilde{\zeta}\left(2 \lambda \tilde{x}_{n}\right)=\inf \left\{\zeta(y): y \in 2 \lambda \tilde{x}_{n}\right\}<\varepsilon$ for $n>N$. Hence there exist $y_{n} \in 2 \lambda \tilde{x}_{n}, n>N$, such that $\zeta\left(y_{n}\right)<\varepsilon$. But $\frac{1}{2} y_{n}-\lambda x_{n} \in \bar{c}$. Arguing as in the proof of Theorem 2.6, with $z_{1}=\frac{1}{2} y_{n}, z_{2}=\lambda x_{n}$, we obtain $\zeta\left(\lambda x_{n}\right) \leq \zeta\left(y_{n}\right)<\varepsilon$ for $n>N$. Hence $\zeta\left(\lambda x_{n}\right) \longrightarrow 0$ as $n \longrightarrow \infty$.

We are indebted to the refree for his kind remarks which enabled to improve the paper, especially in parts concerning the modular $\tilde{\zeta}$.

\section{References}

[1] T.M. JEDRYKA and J. MUSIELAK, Some remerks on $F$-modular spaces, Functiones et Approximatio 2 (1976), 83-100.

[2] J. MUSIELAK, Sequences of finite $\Phi$-variation, Prace Matem. 6 (1961), 165-174 (in Polish).

[ 3 ] J. MUSIELAK, Modular approximation by a filtered family of linear operators, Analysis and Approximation, Preceed. Confer. Oberwolfach, August 9-16, 1980, Birkhäuser Verlag 1981, 99-110.

[ 4 ] J. MusielaK, Orlicz spaces and modular spaces, Lecture Notes in Math. 1034, Springer Verlag, Berlin-Heidelberg-New York-Tokyo 1983. 
[ 5 ] J. MUSIELAK and W. ORLICZ, On modular spaces, Studia Math. 18 (1959), 49-65.

[6] J. MUSIELAK and A. WASZAK, On two-modular spaces, Commentationes Math. 23 (1983), 63-70.

[ 7 ] H. NAKANO, Topology and toptlogical linear spaces, Tokyo 1951.

[ 8] H. NAKANO, Generalized modular spaces, Studia Math. 31 (1968), 439-449.

[9] A. WASZAK, On convergence in some two-medular spaces, General Topology and its Relations to Modern Analysis and Algebra V, Heldermann Verlag, Berlin 1982, 674-682.

Institute of Mathematics,

Adam Mickiewicz University, 